ENCYCLOPEDEE Encyclopédie berbère

BERBERE

$26 \mid 2004$

26 | Judaïsme - Kabylie

\title{
Juifs du Maghreb
}

De la conquête arabe à la décolonisation

\section{J. Taïeb}

\section{OpenEdition}

\section{Journals}

Édition électronique

URL : http://journals.openedition.org/encyclopedieberbere/949

DOI : $10.4000 /$ encyclopedieberbere.949

ISSN : 2262-7197

\section{Éditeur}

Peeters Publishers

\section{Édition imprimée}

Date de publication : 1 mai 2004

Pagination : 3952-3962

ISBN : 2-7449-0452-X

ISSN : 1015-7344

\section{Référence électronique}

J. Taïeb, « Juifs du Maghreb », Encyclopédie berbère [En ligne], 26 | 2004, document J15, mis en ligne le 12 janvier 2012, consulté le 14 décembre 2020. URL : http://journals.openedition.org/

encyclopedieberbere/949; DOI : https://doi.org/10.4000/encyclopedieberbere.949

Ce document a été généré automatiquement le 14 décembre 2020.

(c) Tous droits réservés 


\section{Juifs du Maghreb}

De la conquête arabe à la décolonisation

\section{J. Taïeb}

1 Treize siècles d'histoire, immense durée qui oblige à se polariser sur les faits les plus saillants, les tendances les plus marquantes. Période immense donc, mais jalonnée par des articulations historiques, c'est-à-dire des événements-clefs qui eurent, ipso facto, une postérité.

2 Le premier temps, à notre sens, va de la conquête arabe du Maghreb jusqu'à la fin du $\mathrm{XV}^{\mathrm{e}}$ siècle, grosso modo, la fin de notre Moyen Âge, temps historique lointain où se forge la personnalité du groupe minoritaire. Le deuxième temps couvre les $\mathrm{XVI}^{\mathrm{e}}$, $\mathrm{XVII}^{\mathrm{e}}$ et $\mathrm{XVIII}$ siècles où plus qu'auparavant le Maghreb, et ses communautés juives, est entraîné dans le tourbillon méditerranéen et surtout de plus en plus influencé par les transformations lentes mais fondamentales de l'Europe classique. Le troisième temps enfin, à partir du XIX $x^{e}$ siècle, est marqué par l'irruption fracassante de la modernité occidentale qui aura sur les populations du Nord de l'Afrique, et en particulier les populations juives, des conséquences révolutionnaires.

\section{De « l'âge d'or » à la décadence}

Lorsque dans la deuxième moitié $\mathrm{du}$ viI ${ }^{\mathrm{e}}$ siècle après $\mathrm{J}$.-C, les Arabes abordèrent les rives du Maghreb, ils y trouvèrent, de manière certaine, des communautés juives, depuis longtemps enracinées, et intégrant probablement un certain nombre de prosélytes. L'islamisation de la contrée, patente au viII ${ }^{\mathrm{e}}$ siècle, soumit ipso facto les juifs au statut de la dhimma, connue aussi sous le nom de pacte d'Omar qui aurait été initié par le deuxième calife Omar Ibn el-Khattab en 640 après J.-C. et mis en forme, plus tardivement, par le calife Omar Ibn Abdelaziz.

Ce statut, inégal par nature, laissait au dhimmi ou protégé la libre disposition de ses biens et lui assurait la sécurité. Ce dernier souffrait en contrepartie d'incapacités légales, payait la jizya ou impôt de capitation, discriminatoire par essence et s'engageait à traiter l'islam et les musulmans avec déférence et humilité. 
5 C'est désormais dans ce cadre que va se dérouler l'existence des juiveries du Maghreb avec, dans un premier temps, celui de l'islam classique, une relative tolérance du maître musulman et une vive créativité intellectuelle en symbiose avec la brillante culture arabe d'alors. Temps alcyoniens qui s'abîmeront dans les crises du XII ${ }^{\mathrm{e}}$ siècle.

\section{Brillance et tribulations}

6 Dans les premiers siècles de l'islam (du VIII ${ }^{\mathrm{e}}$ au XII ${ }^{\mathrm{e}}$ siècle après $\left.\mathrm{J} .-\mathrm{C}\right)$, dans l'immense espace allant de l'actuel Irak jusqu'à l'Occident " extrême », le Maroc (et l'Espagne), le pacte fut appliqué avec un certain laxisme car le monde arabo-musulman d'alors, puissant et prospère, traitait sans crispation ses minoritaires, le Maghreb ne faisant, en rien, exception à la règle.

7 Cette tolérance se mua même en une relative bienveillance sous la dynastie fatimide (910-972) en Berbérie orientale. Chiites ismaéliens, adeptes de doctrines ésotériques et régnant sur des populations sunnites, les Fatimides utiliseront volontiers les services des gens du Livre ahl el-kitâb, en l'occurrence les juifs. Presque à la même époque, les Idrissides de Fès (788-959), sans être fondamentalement hostiles aux juifs, furent plus regardants.

8 Les documents de la Gueniza du Caire brossent, pour le Maghreb, de 800 à 1150 après J.$C$, en chiffres ronds, une vaste fresque des communautés juives. Cette période, à bien des égards, apparaît comme une ère de prospérité économique, de brillance intellectuelle avec deux pôles particulièrement actifs, Fès et surtout Kairouan. En matière d'institutions communautaires, les Juifs bénéficient d'une large autonomie et s'émancipent de la tutelle de l'elixarque de Bagdad au début du XI ${ }^{\mathrm{e}}$ siècle. Partout, les juifs des classes aisées accèdent à la culture arabe, parlent, lisent, composent en cette langue, vraisemblablement dès la fin $\mathrm{du} \mathrm{vIII}^{\mathrm{e}}$ siècle. Ces documents attestent aussi du rôle d'espace colonial du monde arabe, joué par le Maghreb, avec un flux régulier et fourni de juifs originaires du Levant et d'Égypte, jusqu'aux débuts du $\mathrm{XI}^{\mathrm{e}}$ siècle. Nouvelle composante ethnique qui vient s'ajouter aux communautés indigènes et peutêtre les submerger, catalysant et accélérant l'arabisation.

Cette longue embellie prit fin au milieu du XII ${ }^{\mathrm{e}}$ siècle avec la conquête des Almohades. Musulmans fanatiques, ils exercèrent, sur les gens du Livre, de sanglantes pressions pour les obliger à abjurer, non sans résultats. Avant 1165 toutefois, il n'y eut pas simultanéité spatiale dans les persécutions mais, après cette date, le judaïsme et le christianisme, officiellement, disparurent du Maghreb. Il est cependant vraisemblable qu'aux marges du Sahara et dans les zones rurales reculées, certains Juifs purent continuer de pratiquer, presque ouvertement, leur foi, alors que les fragiles chrétientés indigènes disparurent ou presque dans la tourmente.

10 Les premiers retours ouverts au judaïsme semblent s'être produits autour de 1230 et se poursuivre dans les décennies suivantes. Ils furent cependant peu nombreux. Pour quelles raisons? Sans doute une partie des descendants des convertis s'étaient-ils sincèrement ralliés à l'islam. Certains hésitaient probablement à revenir au judaïsme car l'abandon de l'islam pouvait être passible de la peine de mort. A cet égard, le massacre de la communauté juive de Fès en 1276 indiquait-il que la population supportait très mal ce retour ? Curieusement, cet épisode almohade, aberrant et grave, n'a laissé, dans la mémoire populaire juive, aucune trace. Cette amnésie s'explique-telle par le petit nombre de retours? Ou bien, les nouvelles communautés juives des 
villes étaient-elles formées de gens venus du désert, de la montagne, des zones rurales reculées où ils avaient continué de pratiquer presque ouvertement leur culte, sans passage à l'islam ? Les deux hypothèses ne sont pas nécessairement contradictoires. En tout état de cause, l'épisode almohade laissa, plusieurs siècles durant, des traces indélébiles.

\section{Crépuscule sur l'Occident musulman}

La période qui va de la fin du XIII ${ }^{\mathrm{e}}$ siècle jusqu'à la fin du Xve siècle est, pour les chétives communautés juives, celle de la misère matérielle et spirituelle et de l'oppression politique. C'en est bel et bien fini de la prospérité économique, de la brillance intellectuelle, de la relative bienveillance des pouvoirs publics. Ce contexte délabré est attesté par les responsa des rabbins d'Alger du XIV et surtout du $\mathrm{XV}^{\mathrm{e}}$ siècle. La population juive d'alors ignore souvent les pratiques élémentaires du judaïsme, fait fréquemment appel à des juges musulmans, adopte dans la vie civile et religieuse des usages islamiques. Sans doute plusieurs générations passées sous le masque musulman expliquent-elles, pour partie, ce déclin tout comme la relative rupture des contacts avec les Juifs d'Europe et du Levant. Dans le même temps, les clercs juifs perdent progressivement la maîtrise de l'arabe classique, si évidente dans les premiers siècles de l'islam. Peut-être, la pression des autorités pour interdire aux Juifs l'usage de l'alphabet arabe est-elle à l'origine de cette perte?

12 Surtout, l'application sévère, mesquine, hostile de la dhimma vire à l'oppression pure et simple. Les Juifs sont astreints ici au port de la rouelle, là à l'adoption de couleurs sombres dans le cadre du ghiyâr ou distinction vestimentaire, peu appliquée aux premiers siècles de l'islam. Discrets, très pauvres (sauf quelques négociants des ports), de tous méprisés, les Juifs du Maghreb central sous les Zayyanides de Tlemcen (1236-1554) et ceux d'Ifriqiya sous les Hafsides de Tunis (1207-1574) évitent-ils, du moins, les persécutions sanglantes. À l'autre extrémité du Maghreb, en revanche, les Mérinides de Fès (1258-1420) et les Wattasides (1420-1548) associent quelquefois des juifs à leur pouvoir mais les violences populaires sont redoutables comme en 1465 à Fès.

Ces temps difficiles furent, dans une certaine mesure, ceux de l'enfermement dans des quartiers spéciaux comme le mellâh de Fès constitué en 1438. Cet enfermement fut-il à l'origine, comme on l'a souvent soutenu, des particularités des parlers arabes des juifs des grandes villes? Ce n'est pas absolument démontré, et sans doute ne faut-il pas négliger d'autres explications comme les influences linguistiques espagnoles à partir du $\mathrm{XVI}^{\mathrm{e}}$ siècle.

14 Ces temps obscurs furent traversés par un événement marquant : l'installation, surtout à Alger, de réfugiés juifs catalans après les massacres de 1391 en Espagne. Plus cultivés et plus riches, les nouveaux venus imposèrent leur liturgie et leurs usages civils à Alger et dans les villes voisines. Ils furent à l'origine d'un renouveau des études religieuses, toujours à Alger et peut-être plus tard à Tunis, alors que Fès aurait toujours compté quelques savants.

Ce crépuscule sur l'Occident musulman n'est en rien un phénomène isolé, il est, dans une large mesure représentatif de l'état du judaïsme mondial et de celui du monde arabe. Dans l'Égypte et la Syrie mameloukes, en effet, l'oppression contre les communautés juives est si forte $d u x{ }^{e}{ }^{e}$ au $x^{e}$ siècle que l'écrasante majorité choisit d'apostasier ou de fuir, vidant le judaïsme égyptien, en particulier, de ses forces vives. 
En Europe, chassés des foyers d'Occident (France, Angleterre), partout discriminés voire massacrés, les Juifs ne font que survivre, menant une existence rétrécie à l'ombre du ghetto, le judaïsme espagnol faisant, pour peu de temps encore, exception à la règle.

\section{0-1800 : le nouveau Maghreb}

16 À la fin du Xve siècle, à la veille de l'arrivée des Juifs ibériques, le judaïsme maghrébin, des sables de la grande Syrte aux rives de l'Atlantique, comptait sans doute moins de 100000 adeptes, quelque $2 \%$ de la population totale. Cette population juive comportait une forte composante urbaine avec une concentration importante dans les grandes villes de l'intérieur comme Fès, Meknès ou Constantine et les grands ports, Alger, Tunis, voire Tripoli. Elle comprenait aussi, on le sait moins, une minorité rurale non négligeable, surtout au Maroc: Juifs montagnards de l'Anti-Atlas, du Haut Atlas et du Draa, à l'époque, probablement berbérophones, juifs semi-nomades des steppes tunisiennes et de manière plus évidente de la région de Constantine, vieilles implantations montagnardes dans le Nefoussa et le Gharyan au sud de Tripoli.

Ces Juifs du Maghreb, depuis la disparition (du XII ${ }^{\mathrm{e}}$ au XIV ${ }^{\mathrm{e}}$ siècle) des derniers résidus chrétiens, étaient désormais les seuls dhimmi, pour le meilleur et pour le pire, contrairement au Machrek où perdurait de fortes chrétientés indigènes. Autre originalité de l'occident musulman, le triomphe quasi total du sunnisme, dans sa variante malékite, relativement rigoriste et sévère à l'égard des gens du Livre.

18 Cet univers stable et quelque peu stagnant allait être bouleversé, entre 1492 et 1600-1610, par des événements marquants de l'histoire générale comme l'achèvement de la reconquista en Espagne en 1492 et le repli subséquent de musulmans grenadins vers le Maghreb, les tentatives ibériques d'installation dans les ports maghrébins, l'arrivée en 1609-1610 des Morisques chassés d'Espagne et, par dessus tout, au XVI siècle, la présence ottomane en «Libye, Tunisie et Algérie » coupant en deux le monde $\mathrm{du}$ « berbéro-islam » et isolant le royaume chérifien.

19 Les communautés furent affectées par ces événements et par d'autres tenant spécifiquement à l'histoire juive comme l'expulsion des juifs d'Espagne de 1492. Cette nouvelle donne allait certes transformer leur destinée, mais, au-delà, ces temps nouveaux présentent une claire distinction des époques avec un $\mathrm{xVI}^{\mathrm{e}}$ siècle agité et relativement confus, un XvII siècle où se mettent en place les structures politiques, économiques et socio-culturelles, du Maghreb moderne, structures subtilement modifiées au XVIII ${ }^{\mathrm{e}}$. De ces changements globaux, les Juifs furent partie prenante.

\section{Un siècle nerveux}

La poursuite de la reconquista en terre d'Afrique eut, pour les communautés juives, des conséquences négatives. Les Espagnols, entre autres ports, prirent Tripoli en 1511, Tunis en 1535, Tlemcen en 1543. La population, dont les juifs, y fut massacrée, vendue comme esclave, contrainte à la fuite. Les communautés ne se reconstituèrent qu'à la fin $\mathrm{du} \mathrm{XVI}{ }^{\mathrm{e}}$ siècle lorsque les Turcs reprirent la plupart de ces cités.

21 Autre événement d'importance, l'arrivée des juifs chassés d'Espagne en 1492, puis, en rangs moins serrés, celle des exilés du Portugal en 1496-97. Ces réfugiés, dans leur grande majorité, choisirent de s'établir au Maroc où leur installation déclencha de 
longs conflits avec les juifs indigènes (tochavîm en hébreu, beldiyyîn en arabe). Nombreux, influents, riches, cultivés, les nouveaux venus finirent par imposer leurs usages religieux et leur liturgie, tout au moins dans le Nord-Est et les grandes cités de Salé, Meknès et surtout Fès. Mais ils échouèrent dans leurs tentatives d'éradiquer la polygamie chez les indigènes. Leur victoire fut cependant si complète, en matière liturgique, qu'au $\mathrm{xx}^{\mathrm{e}}$ siècle, dans la ville de Fès, il ne subsistait qu'une seule synagogue pratiquant le vieux rite, celle dite des Fassi. Le phénomène n'est pas sans rappeler ce qui se produisit à Alger après 1391 où les Catalans imposèrent, progressivement, leur rite, celui des mellâhîn (les gens venus par le mer en arabe?) au point que la liturgie des hâarriyyîn (les gens de la ḥ̂ara d'Alger, les indigènes) disparut à la veille de la conquête française de 1830 .

\section{Au XVII ${ }^{\mathrm{e}}$ siècle, une nouvelle donne}

Politiquement, la présence ottomane se marqua par l'installation de trois régences, celle de Tripoli, de Tunis et d'Alger; économiquement, par le recul de la puissance bédouine et la renaissance villageoise, surtout en Tunisie. Renaissance en partie liée à l'arrivée des Morisques au début du XvII ${ }^{\mathrm{e}}$ siècle et qui modifia quelque peu la vie rurale tandis que la course, certes active avant le XVII ${ }^{\mathrm{e}}$ siècle, connut un considérable essor, enrichissant les particuliers et l'État, à Rabat, Salé, Alger, Tunis et Tripoli. Dans le même temps, se développait avec l'Europe le négoce maritime, Livourne et Marseille jouant un rôle-clef dans les courants commerciaux.

Sans être en rien monopole, l'action des Juifs dans le rachat des captifs chrétiens dans la course était importante, tout comme leur activité commerciale avec Livourne. Dans le négoce comme dans le rachat, le rôle des Juifs de Livourne, et de loin, l'emportait sur celui des juifs indigènes. Les premiers étaient, en fait, des marranes hispano-portugais, ou plus précisément d'anciens marranes, passés par le port toscan après son ouverture aux «nouveaux chrétiens", à l'extrême fin $\mathrm{du} \mathrm{XvI}^{\mathrm{e}}$ siècle, et établis à Tripoli, plus encore à Alger et surtout à Tunis, au tout début du XVII siècle. Cette activité portuaire suscita, surtout au centre et à l'est du Maghreb, un certain afflux de Juifs issus des petites villes et des zones rurales, exode dont bénéficièrent trois cités : Tripoli, Tunis et Alger.

L'arrivée des Juifs ibériques entraîna aussi, au Xvi ${ }^{\mathrm{e}}$ siècle, une effervescence mystique, l'aspect cardinal de cette effervescence tournant autour de la cabbale, d'abord au Maroc puis graduellement dans tout le Maghreb. À partir du début du XvII ${ }^{\mathrm{e}}$ siècle, on réactiva, de par la volonté des cabbalistes, des dévotions quelque peu oubliées. Il s'agissait des mélodies mélancoliques dites baqqachôt, vigoureuse synthèse entre poésie hébraïque et plain-chant andalou qui, des générations durant, essentiellement au Maroc, représenta, par excellence, et l'art musical et la ferveur religieuse. Toujours au XVII ${ }^{e}$ siècle, la multiplicité des échanges avec l'Europe et surtout Livourne fit pénétrer l'imprimé hébraïque au Maghreb, diffusant le savoir et élargissant le nombre des clercs, et là encore, le rôle des Livournais d'Alger et de Tunis fut considérable par leur mobilité, leur ubiquité, leur haut niveau culturel. 


\section{Le fécond XVIII ${ }^{e}$ siècle}

Après 1730, la course en Méditerranée s'essoufflant, les finances publiques et privées d'Alger entrent en crise tandis que Tripoli et plus encore Tunis s'en tirent bien mieux en développant, à grande échelle, le commerce maritime avec l'Europe, Marseille l'emportant désormais sur Livourne. Au Maroc, l'essor du commerce maritime au loin fut plus tardif qu'ailleurs au Maghreb et fit la fortune des « commerçants du sultan », tujjâr es-solțân, musulmans andalous, Européens et surtout Juifs, installés dans le nouveau port d'As-sawira (Mogador) et voués au négoce atlantique avec les puissances protestantes.

En matière institutionnelles, l'événement le plus symbolique fut le "schisme », en 1710, à Tunis, des Juifs livournais ou Grâna qui se séparèrent unilatéralement des Tunisiens ou Twânsa en créant des institutions communautaires séparées, «schisme » finalement reconnu par les Twânsa en 1741, choc culturel majeur entre arabisés, Must'arebîn, et fiers juifs ibériques, fait atypique, car, partout ailleurs au Maghreb, les Espagnols s'étaient emparés des leviers de commande communautaires. Pourquoi à Tunis, et à Tunis seulement, les indigènes refusèrent-ils d'abdiquer?

Le $\mathrm{XVIII}^{\mathrm{e}}$ siècle fut aussi par excellence celui de l'effervescence urbaine, de l'expansion spatiale des quartiers juifs, de l'exode rural. Si la population juive d'Alger, rançon du déclin économique de la ville-État, régressa lentement, tout au long du siècle, si les Juifs, dans quelques cités marocaines, comme Fès, Meknès, Marrakech, étaient enfermés depuis plusieurs générations dans le mellâh ceint de murailles; on notait, un peu partout, la création de nouvelles communautés dans les ports comme Mogador, au Maroc, Sfax et Mahdia, en Tunisie, Oran, reconquise, en 1792, sur les Espagnols par le dey d'Alger. Dans certains centres, la population juive, en nette croissance, déborda son habitat traditionnel, celui de la ḥâra (le quartier juif). À Tunis elle s'étendit vers l'est, dès le début $\mathrm{du} \mathrm{xVII}^{\mathrm{e}}$ siècle et plus encore au $\mathrm{xVIII}^{\mathrm{e}}$ siècle. À Tripoli, l'espace compris entre le petit quartier, hâara sghîra, et le grand quartier hâara kbîra fut lentement investi. L'immigration vers les grandes villes, exode rural pour l'essentiel, est d'ailleurs encore présente dans la mémoire populaire, essentiellement par le canal de l'onomastique.

Ce fertile XVIII ${ }^{e}$ siècle fut encore celui d'une considérable activité éditoriale des rabbins locaux, avec le plus souvent une littérature décisionnaire et des gloses talmudiques, généralement imprimées à Livourne, encore et toujours Livourne! S'enracinèrent alors des lignées de savants et d'érudits comme les Abensur de Fès, les Benattar présents dans plusieurs cités du Maroc, les Berdugo et Toledano de Meknès, les Borgel de Tunis et nous en oublions. Dans ce même temps, autre phénomène d'ouverture et de mobilité, se multiplièrent les voyages des rabbins émissaires de Palestine faisant circuler les idées, les livres, les informations, les airs liturgiques.

Tous faits de société éminemment novateurs, élargissant et approfondissant certes les tendances déjà présentes au XVII ${ }^{e}$ siècle mais multipliant les innovations majeures comme la production, in situ, d'une considérable littérature rabbinique.

1500-1800, trois siècles de mutations ou, plus modestement, de changements lents mais réguliers et significatifs. S'insinue alors, au Maghreb, une première modernité, ou une prémodernité, antérieure à la Révolution industrielle et à l'intrusion massive de l'Occident. Prémodernité catalysée, dans une large mesure, par l'arrivée de Juifs ibériques au Maghreb, soit directement après 1492-1496, soit plus tardivement par le canal de Livourne. Prémodernité qui affecte, aussi et surtout, les communautés juives 
d'Europe, déjà quelque peu pénétrées par les Lumières et en plein essor démographique après 1700. Les communautés juives du Maghreb s'insèrent donc dans les tendances lourdes du judaïsme mondial d'alors.

Par rapport cependant au Levant ottoman, plus précisément, par rapport au monde judéo-espagnol de Grèce et du Bosphore, d'une part, et aux communautés juives arabophones d'Égypte et du croissant fertile, d'autre part, nos Juifs des échelles de Barbarie et même du Maghreb intérieur sont affectés d'un dynamisme plus grand. Après, en effet, un $\mathrm{xVI}^{\mathrm{e}}$ siècle relativement prospère et une relative tolérance d'un maitre turc, le XVII ${ }^{\mathrm{e}}$ siècle, au Levant, voit un durcissement du statut des Juifs en même temps que les chrétiens locaux, aidés par la sollicitude de l'Europe et par l'ouverture d'écoles modernes, concurrencent victorieusement les Juifs dans le commerce extérieur et l'appareil d'État. Les communautés juives du Levant sont, en même temps, psychologiquement épuisées, à la fin du XVII ${ }^{e}$ siècle, par le désastreux épisode du faux messie Chabbâtay Zvi, alors que celles du Maghreb le sont beaucoup moins, dans un contexte où l'absence de chrétientés indigènes fait des Juifs indigènes, et surtout des Livournais, les intermédiaires obligés vis-à-vis de l'Europe et les vecteurs du changement social.

\section{0-1980. L'intrusion fracassante de la modernité}

Un siècle et demi de considérables bouleversements, de mutations inouïes, impensables avant 1830. Le changement social, dans toutes ses manifestations, se fait alors extrêmement rapide, continu, déstabilisateur, acculturant. Dans cet univers où tout dorénavant bouge, des articulations historiques avec de sensibles retombées sont cependant aisément perceptibles. Elles serviront de balises dans la trame événementielle qui va suivre.

\section{Les Juifs du Maghreb à la veille de 1830}

Pourquoi d'abord ce repère de 1830 ? Tout simplement parce qu'il marque, avec la prise d'Alger par les Français, le début du processus de modernisation dont il a été question plus haut.

Vers 1820-1830, on dénombrait, très grossièrement, 140000 Juifs au Maghreb (un peu moins de $2 \%$ de la population totale), dont vraisemblablement quelque 10000 en Libye, 30000 en Tunisie, 25 à 30000 en Algérie, 70000 ou plus au Maroc. Cette population souffrait partout d'un statut juridique très diminué mais il faut honnêtement distinguer entre les trois régences ottomanes et le royaume chérifien. Dans les trois premières, dominait la dhimma classique c'est-à-dire un statut d'oppression, sans excès sanglants de la populace ou des autorités, contrairement au Maroc.

Linguistiquement arabisée depuis de très longs siècles, la collectivité comptait cependant en son sein quelques minorités non arabophones: les hispanophones du nord du Maroc, en place depuis 1492, pratiquant un dialecte judéo-espagnol dit hakètiya et le groupe de langue et de culture berbère du Haut Atlas central au Maroc, dernier bastion possible d'une aire jadis étendue à tout le Haut Atlas, à l'Anti-Atlas, à la plaine du Sous et au Draa. Mais ces populations auraient été arabisées au XVII et surtout au 
$\mathrm{XVIII}^{\mathrm{e}}$ siècle par des Juifs arabophones, porteurs de cette langue prestigieuse et semble$\mathrm{t}$-il originaires d'Illigh, Demnat et surtout Ifrane.

Socialement et géographiquement diversifiées, les communautés appartenaient toutes au monde de la tradition, "ce monde que nous avons perdu ", selon l'expression de l'historien britannique Peter Laslett. Croyances traditionnelles, où se mêlaient religion et superstitions, société traditionnelle, avec une mince élite de clercs et de négociants, une masse pauvre de boutiquiers et d'artisans, un grand nombre de misérables, mentalités traditionnelles sans remise en cause sacrilège de l'ordre du monde, social ou politique. Certes, quelques frémissements étaient apparus à l'extrême fin du XVIII siècle avec les Lumières et la Révolution française. Certes encore, certains, qui avaient voyagé, savaient que les Juifs de Bordeaux, Amsterdam et Livourne étaient des hommes libres, mis sur un pied d'égalité avec les chrétiens. Certes enfin, quelques-uns, sans doute, aspiraient à sortir leurs coreligionnaires de leur situation diminuée. Mais ces constats et ces aspirations demeuraient très minoritaires, presque marginaux.

\section{La prise d'Alger et ses retombées}

Juillet 1830, "Alger la bien gardée " tombe entre les mains des Français. Dans les décennies qui suivent, les troupes françaises étendent leur emprise sur le reste du pays et dans ce temps même, les communautés juives de l'ex-régence sont entraînées dans un extraordinaire tourbillon bouleversant leur quotidien et transformant leur statut politique, avec l'entrée dans le droit commun en matière juridique et l'abolition de facto de la dhimma. Dans le même temps s'effondre rapidement l'autonomie communautaire. La "nation juive ", corps politique distinct, n'existe plus dès 1845 avec la mise en place des consistoires sur le modèle français. Le 24 octobre 1870 ensuite, la promulgation du décret Crémieux transforme en citoyens français les Juifs indigènes. Ce décret allait soulever des oppositions passionnées au sein de la population européenne de la colonie. Opposition qui culmina lors de la crise anti-juive de 1897-98. Mais le gouvernement de Paris, en dépit de l'agitation, refusa l'abrogation. Les ordonnances du 28 février 1841 et du 26 septembre 1842 confièrent les procès entre Israélites, y compris ceux relatifs au statut personnel (héritages, mariages, divorces), aux tribunaux français, mais ce statut lui-même disparut avec l'entrée dans la citoyenneté française après 1870.

Économiquement, socialement, culturellement, la population accédait, graduellement d'abord, à marche forcée ensuite, à la modernité. Les juifs multiplièrent les boutiques dans les quartiers européens avec une clientèle plus exigeante et au niveau de vie plus élevé que celui des clients traditionnels musulmans ou juifs. Pour la plupart des communautés, la mobilité sociale ascendante devient la règle tandis que l'accroissement démographique se faisait spectaculaire, rompant avec la stagnation séculaire. On comptait quelque 26000 Juifs en Algérie, à la veille de la conquête française, sans doute 52000 en 1880 et 96000 en 1913-14. Culturellement, enfin, le fait majeur fut le lent abandon de l'arabe et l'accès à la langue française. Le décret Crémieux renforça et accéléra ces tendances après 1870, mais cette modernité multiforme laissait intact ou presque le monde de la tradition dans l'est et le sud du pays, tout particulièrement au Mzab et à Constantine. 


\section{Hors d'Algérie. Dissemblances et analogies}

39 La présence française en Algérie entraîna un regain inattendu d'activisme turc avec la reprise en main de la régence de Tripoli en 1835. De la sorte, les communautés juives bénéficièrent du hatti humayûn de 1856 qui, sans abolir explicitement la dhimma et sans faire disparaitre les autonomies communautaires, établissait une relative égalité entre musulmans et non-musulmans au sein de l'empire ottoman, égalité mal acceptée par les populations musulmanes. Dans cette logique de sécularisation, les tribunaux rabbiniques furent cantonnés aux problèmes de statut personnel.

Dans la Tunisie voisine, la promulgation, en 1857, du pacte fondamental allait dans le même sens que le réformisme ottoman de 1856 et là aussi les populations musulmanes réagirent défavorablement. Comme en Libye enfin, les tribunaux rabbiniques se virent limités aux questions du statut personnel.

41 Au Maroc, où la situation des Juifs était la plus délabrée, le tournant de 1862-63 fut significatif. L'Alliance israélite universelle (AIU) ouvrit en effet sa première école à Tétouan en 1862 et, l'année suivante, le voyage au Maroc du philanthrope Moses Montefiore, sans résultat concret dans l'immédiat, attira toutefois l'attention internationale sur le sort des Juifs marocains. Au royaume chérifien cependant, l'archaïque statut de la dhimma perdura vigoureusement jusqu'à la fin du XIX siècle. Ensuite, et ensuite seulement, sans avoir jamais été formellement abrogé, il commença à s'effondrer dans les villes de la côte où les juifs pouvaient bénéficier de l'appui des consuls européens et de l'Alliance israélite. Mais dans l'intérieur, cette liberté nouvelle, où cette arrogance nouvelle, des Juifs de la côte, entrainait une détérioration du sort de leurs coreligionnaires et également de véritables « pogroms » un peu partout, à Demnat mais aussi à Casablanca (1907) et à Fès (1912).

Globalement donc, de profondes différences semblent séparer les Juifs d'Algérie de ceux des autres contrées après 1830. Dans le premier pays, le changement institutionnel fut brutal et rapide, ainsi que le changement social (surtout après 1870) bousculant, déstabilisant, un monde traditionnel. Ailleurs, les évolutions furent plus lentes, moins brutales. Ailleurs aussi, les protections des consuls européens furent patentes après 1840 et l'action scolaire et politique de l'AIU particulièrement efficace (après 1860). En Algérie en revanche, l'école républicaine scolarisant les jeunes Juifs, l'œuvre de l'AIU fut plus spécifique et plus limitée. Quant aux protections des consuls européens, elles n'avaient pas lieu d'être là où flottait le drapeau français.

Mais au-delà de ces divergences, partout avec ou sans pouvoir colonial, les communautés juives entamèrent une marche régulière vers l'égalité des droits, la sécularisation (plus nette en Algérie) et le recul de l'autonomie communautaire (également plus net en Algérie). Partout enfin, l'émancipation des Juifs se heurta à de tenaces oppositions populaires, à des réticences dans les sphères gouvernementales en Libye et surtout en Tunisie, à des refus intransigeants des pouvoirs publics au Maroc.

\section{Le triomphe colonial}

1881 traité du Bardo, qui instaure le protectorat français en Tunisie, 1911 début de la conquête italienne en Libye, 1912 protectorat français sur le Maroc. 
45 À la veille de la Grande Guerre, comme un fruit mûr, le grand Maghreb était, tout entier, subjugué par l'Europe. Mais les sociétés juives, désormais en contact direct avec la France, l'Italie (en Tunisie) et l'Espagne (au Maroc), n'étaient plus, tout à fait, des sociétés traditionnelles, pénétrées qu'elles avaient été par la lente infiltration, depuis deux générations, des idées, des hommes, des capitaux, des marchandises venus d'Europe. Dissemblance donc d'avec les communautés d'Algérie de 1830-1840 fascinées et affolées par l'irruption d'un monde nouveau. C'est donc dans un univers déjà quelque peu transformé que se fixa l'empreinte indélébile de la colonisation.

Ce temps colonial, relativement court (1881-1956 en Tunisie, 1911-1952 en Libye, 1912-1956 au Maroc), sauf en Algérie (1830-1962), vit s'amplifier et s'accélérer dans les trois premiers pays le processus de modernisation déjà enclenché avant la colonisation avec des changements institutionnels, comme l'entrée globale des communautés juives dans le droit commun, avec cependant des limites précises: maintien du statut personnel, d'une certaine autonomie communautaire, absence d'accession à la citoyenneté française, italienne ou espagnole, limites inexistantes, depuis longtemps, en Algérie. Mais c'est surtout en matière de changement social que les bouleversements furent de plus en plus significatifs : rapide diffusion du français, de l'espagnol, de l'italien, scolarisation de masse dans les écoles publiques ou celles de l'AIU, émancipation croissante des femmes, etc. En matière démographique les changements furent aussi considérables. On passa ainsi de moins de 20000 Juifs en Libye vers 1911 à 37 ou 38000 en 1946-47, d'environ 25000 en Tunisie vers 1881 à 105-110 000 en 1946-47, de quelque 115000 au Maroc en 1912 à 250 ou 270000 à la fin des années quarante. Tandis qu'en Algérie de 96000 personnes en 1913, on arrivait à 110000 en 1931 et 125000 en 1946-47, accroissement déjà plus lent, signe d'un contrôle des naissances apparu vers 1900, typique de la modernité occidentale.

Cette modernité toutefois rencontrait jusqu'à la fin des années quarante et au-delà de puissants môles de résistance: au sein des masses populaires nettement plus nombreuses que les classes moyennes au Maroc, en Tunisie, en Libye et dans l'est de l'Algérie, dans les zones rurales et montagnardes restées traditionnelles comme aux marges du Sahara.

\section{La fin d'un monde}

48 La création de l'État d'Israël et la décolonisation allaient, en un peu plus de deux décennies, détruire de très anciennes communautés. Bouleversement inouï, impensable une génération plus tôt. De cette accélération de l'histoire, la trajectoire fut simple. Dès la fin des années quarante et le début des années cinquante, en un temps où la présence française en Afrique du Nord ne semblait pas menacée, des foules nombreuses prirent le chemin de la terre sainte, surtout issues du Maroc et de la Tunisie, les communautés les plus traditionnelles. Ces départs n'étaient en rien une originalité, ils affectaient, à la même époque, de manière massive, les communautés juives de l'Orient arabe : Égypte, Irak, Syrie, Yémen. Mais dans ces derniers pays, les menaces physiques et politiques nées du conflit israélo-arabe jouèrent un rôle considérable, le même phénomène se retrouvant en Libye où l'accession à l'indépendance en 1952 fut précédée de graves désordres anti-juifs en 1946 et 1948. Dans l'Afrique du Nord française, en revanche, les départs s'expliquaient essentiellement par l'attraction politique de l'État juif, attraction 
fortement teintée d'aspirations religieuses et par le souci, chez les plus pauvres, d'améliorer leur situation économique dans un pays neuf.

L'indépendance venue au Maroc, Tunisie puis Algérie, les départs devinrent plus massifs au point de vider de l'essentiel de leur substance les communautés des trois pays, dès 1962-63 en Algérie, douze ans plus tard au Maroc et en Tunisie. Deux séries de causes se croisaient quant à l'origine de cette émigration de masse. Les unes directement liées au conflit israélo-arabe, surtout après 1967, les autres à la décolonisation et aux difficultés politiques, économiques et culturelles qu'elle engendrait.

Globalement donc, vers 1830, en dépit des multiples évolutions des XVII ${ }^{e}$ et XVIII siècle, les sociétés juives du Maghreb, demeurent des sociétés traditionnelles. Ce monde de la tradition fut brutalement subverti, après cette date d'abord et surtout en Algérie, plus tardivement (vers 1850-60), plus lentement, moins profondément ailleurs.

Phénomène total, cette subversion fut une tendance lourde bousculant, partout, les structures politiques sociales et économiques du groupe, transformant sa vision du monde. Cette évolution ne se borna pas à l'Afrique du Nord, elle gagna les autres périphéries d'Occident où justement vivaient la majorité des juifs : Europe orientale, Balkans, Orient arabe. Par rapport aux communautés les plus proches culturellement, celles du Machrek (Irak, Syrie, Égypte), de langue et de culture arabes, celles du Bosphore et du sud des Balkans, de langue et de culture judéo-espagnoles, les juifs du Maghreb se trouvaient cette fois-ci, contrairement aux XVII ${ }^{e}$ et $\mathrm{XVIII}^{\mathrm{e}}$ siècles, en évidente concordance de phase avec la même pénétration massive de l'Occident, le même rôle dévolu à l'Alliance israélite universelle, etc. Dans cette course à la modernité, le cas de l'Egypte est à la fois très particulier et paradigmatique, poussant à l'extrême les grandes lignes de l'évolution plus haut décrite. Démographiquement insignifiante, économiquement et culturellement marginale vers 1810-1820, la communauté juive fut une pure création de la modernité dans le second XIX ${ }^{e}$ siècle, passant de 7000 personnes vers 1840 à presque 40000 en 1907, s'approchant des 60000 en 1914 pour dépasser 65000 vers 1947-48, recevant notamment massivement les immigrants de toute la Méditerranée (Maghreb, Bosphore, Grèce, Syrie), acquérant une grande visibilité humaine et économique dans les grandes villes comme Le Caire et Alexandrie. En bref, un miroir grossissant de ce qui dans le même temps se passait à Casablanca, Alger, Tunis et Tripoli.

Que retenir au terme de ce long périple? Notons, tout d'abord, que la présence juive remonte, en ces contrées de Berbérie, à une lointaine antiquité. Y avait-il des Hébreux parmi les fondateurs de Carthage ? C'est possible mais non prouvé.

Y eut-il conversion de Berbères au judaïsme, aux premiers siècles de l'ère chrétienne ? C'est vraisemblable, probable, mais présomption ne signifie pas certitude.

On se meut, en revanche, sur un terrain plus solide en affirmant la présence de Juifs en Berbérie orientale (y compris la Cyrénaïque), aux premiers siècles du christianisme, Juifs probablement venus de Terre sainte. On peut aussi considérer comme certaine l'arrivée, aux temps de l'islam classique, de nombreux transfuges d'Orient (Egypte et Irak en particulier). Certaine également, l'arrivée des vagues ibériques en 1391, 1492 et 
au-delà ; certaine enfin, l'installation des Livournais à Tripoli, Alger et surtout Tunis dès le début $d u \mathrm{XVII}^{\mathrm{e}}$ siècle. Lointaine, la présence juive est aussi une présence diversifiée, de par l'origine ethnique et géographique de ses composantes.

Retenons, ensuite, que cette longue histoire en terre d'islam, dans le contexte particulier de la Berbérie, quelque peu différent de celui du Machrek, s'articule en trois temps. Celui de l'islam classique tolérant, prospère, intellectuellement fécond mais qui s'abîme au XII ${ }^{e}$ siècle dans le déclin et l'intolérance. Celui des temps modernes, extravertis et marqués par des changements lents et continus. Celui enfin des ruptures fondatrices du XIX ${ }^{e}$ siècle, fondement de la modernité, modernité largement exogène, révolutionnaire, déstabilisante, émancipatrice, qui aboutira à la disparition de l'objet même de notre étude au siècle suivant.

En l'espace de trois ou quatre générations, se transformeront, en profondeur, et le quotidien et la vision du monde du groupe considéré. Mutations multiples et qui amèneront, par exemple, cette collectivité, depuis des siècles désarmée, à la découverte des armes.

\section{BIBLIOGRAPHIE}

ABITBOL M., Le passé d'une discorde. Juifs et Arabes du VII ${ }^{\mathrm{e}}$ siècle à nos jours, Paris, Perrin, 1999.

BRUNSCHVIG R., La Berbérie orientale sous les Hafsides. Des origines à la fin du XV siècle, Paris, Librairie d'Amérique et d'Orient, Adrien Maisonneuve, tome I, 1940, tome II 1947.

GOITEN S.D., A Mediterranean society. The jewish communities of the Arab world as portrayed in the documents of the Cairo gueniza, Berkeley-Los Angeles, London, University of California press, tome I 1967, tome II 1971, tome III 1978, tome IV 1983.

HIRSCHBERG H. Z., A history of the jews in North Africa, Leiden, E.-J. Brill, tome I 1974, tome II 1981.

IANCU-AGOU D., « Une communauté juive dans le Sud Algérois. Djelfa 1852-1962 », in Communautés juives des marges sahariennes du Maghreb, édité par Michel Abitbol, Jérusalem, Institut Ben Zvi, université hébraïque, 1982, pp. 161-190.

PLANHOL X. de, Minorités en Islam. Géographie politique et sociale, Paris, Flammarion, coll.

«Géographes », 1997.

STILlman N. A., The Jews of Arab land. A History and source book, Philadelphia, The jewish publication society of America, 1979.

TAÏEB J., Sociétés juives du Maghreb moderne. Un monde en mouvement (1500-1900), Paris, Maisonneuve et Larose, 2000.

ZAFRANI H., Juifs d'Andalousie et du Maghreb, Paris, Maisonneuve et Larose, 1996. 
INDEX

Mots-clés : Almohades, Al-Andalus, Arabes, Catalogne, Chiisme, Dhimmî, Espagne, Fatimides, Israël, Maroc, Mérinides, Morisques, Moyen Âge, Portugal, Protectorat, Reconquista 